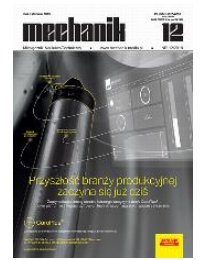

\title{
Evaluation of coordinate measurement uncertainty of parallelism of axes by means of sensitivity analysis method
}

\author{
WOJCIECH PŁOWUCHA * \\ * Dr inż. Wojciech Płowucha, wplowucha@ath.bielsko.pl, https://orcid.org/0000-0003-0446-9052 - Akademia Techniczno-Humanistyczna \\ w Bielsku-Białej, Laboratorium Metrologii, Bielsko-Biała, Polska
}

On the examples of measurement of three variants of the parallelism of the connecting-rod centre axes the theoretical basis of a new method for estimating the uncertainty of coordinate measurements is presented. This is a continuation of the article "Estimation of coordinate measurement uncertainty by means of sensitivity analysis - theoretical background" (Mechanik 11, 2018). In the first variant, the tolerance zone is in the form of a cylinder, in the other two it has the form of a pair of planes. In the examples presented, uncertainty budgets contain six or nine input factors. In all cases, when the axes of the measured holes are parallel to one of the machine axes, only two input factors affect the uncertainty of the measurement of parallelism deviation.

KEYWORDS: measurement uncertainty, sensitivity analysis, coordinate measurements

\section{Introduction}

Basics and important assumptions of the new method developed at ATH for estimating the uncertainty in coordinate measurements are presented in publications [1, 2]. In [1], examples of uncertainty of measuring the circle radius and circle arc radius are also given. The term "sensitivity analysis method" in the title was taken from the technical specification ISO/TS 15530-1: 2013 [3], which in addition to this method, distinguishes the method using the reference object [4] and simulation.

The approach directly resulting from the recommendations of the GUM guide [5] was used: starting point is the measurement model in the form of a formula for the measured characteristics, and the sensitivity coefficients are values of partial derivatives calculated from the measurement model. Despite relatively simple form of the formulae for the individual geometrical characteristics of the measured workpieces, formulae for partial derivatives are quite complex and computer aided calculations are necessary. The author uses the Maplesoft Maple 2018 software. A spreadsheet containing the calculations can be found on the website of the ATH Laboratory of Metrology in the "Download" tab (www.lm.ath.bielsko.pl).

This publication presents three examples of estimating the measurement uncertainty of the parallelism of the connecting rod axes, corresponding to the three tolerance methods found in ISO 1101 [6]. These are the following cases:

- cylindrical tolerance zone,

- in-plane tolerance,

- out-of-plane tolerance.

Two of them were analysed in detail. For the third, most complex, only the model and results are given. The example contains all details enabling recalculating for different data - both dimensions of the workpiece and accuracy of the CMM. Like in the previous paper, it was assumed that the standard measurement uncertainty of coordinates' differences is calculated from the formula:

$$
u_{x i}=E_{L, M P E} / 3
$$


and the maximum permissible error of length measurement of the CMM used is given by:

$$
E_{L, M P E}=(2+0,004 L) \mu \mathrm{m}
$$

in which: $L$ - measured length [mm].

\section{Axis parallelism deviation - cylindrical tolerance zone}

In Fig. 1 there is an example of the specification of tolerance and measurement model of the axes' parallelism deviation for a cylindrical tolerance zone.

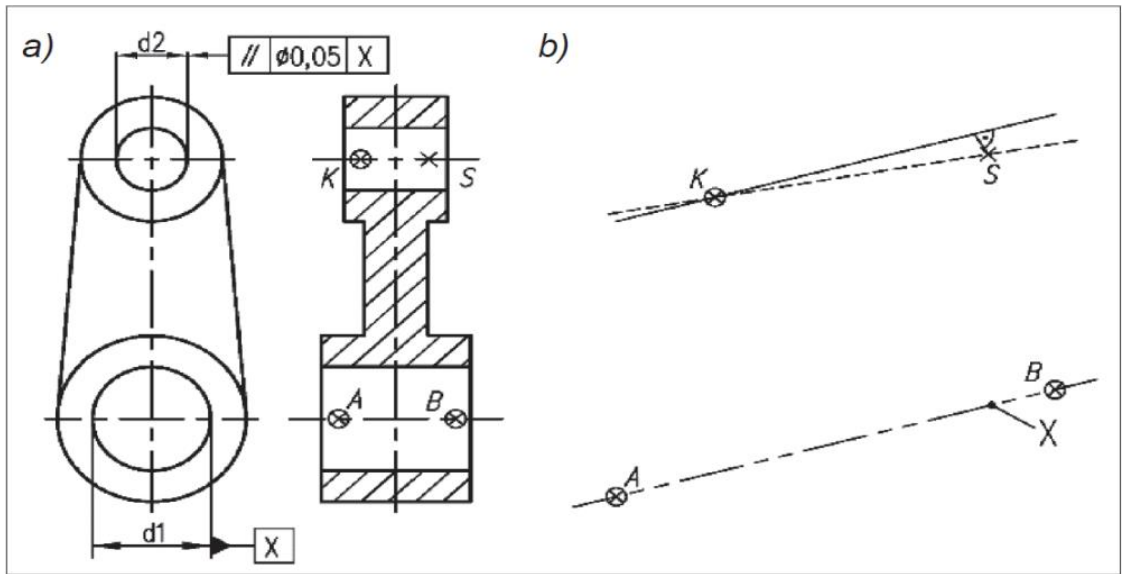

Fig. 1. Parallelism of axes in a case of cylindrical tolerance zone: $a$ ) example drawing with characteristic points, $b$ ) model for uncertainty evaluation

The parallelism of axes tolerance zone according to ISO 1101 is a cylinder with a diameter equal to the tolerance value. The parallelism of axes deviation is the smallest diameter of the cylinder, the axis of which is parallel to the axis $A B$ and which covers all axis points of the toleranced element. In the presented model, these are points $K$ and $S$, i.e. the deviation is equal to the distance $l$ of the point $S$ from the straight line containing the point $K$. The uncertainty of the measurement of parallelism deviation is equal to the uncertainty of measurement $u_{1}$ of distance $l$.

General formula for the distance $l$ of the point $S$ from the line $p$ defined by the point $P$ and the unit vector $v$ has the form:

$$
l(S, p)=|(P-S) \times v|
$$

In the measurement model, the straight line $p$ is represented by one point and is parallel to the straight line defined by two points.

In this case, point $K$ will be used as point $P$ in formula (3), and the unit vector $v$ will be determined as:

$$
v=\frac{B-A}{|B-A|}
$$

Finally, a measurement model is obtained in the form:

$$
l=\left|K S \times \frac{A B}{|A B|}\right|
$$

where: $A B=\left[a b_{1}, a b_{2}, a b_{3}\right], K S=\left[k s_{1}, k s_{2}, k s_{3}\right]$.

Distance $l$ is a function of six coordinates' differences (vector components), thus the combined uncertainty of measurement $u_{l}$ is the geometric sum of six elements:

$$
u_{l}=\sqrt{\sum_{i=1}^{6}\left(\frac{\partial l}{\partial x_{i}} u_{x i}\right)^{2}}
$$


It is worth noting that the distance between the toleranced element and the datum does not occur in the model. In the scalar notation, the formula for distance $l$ (measurement model) is of the form:

where:

$$
l=\frac{L}{M}
$$

$$
\begin{aligned}
L & =\sqrt{L_{1}^{2}+L_{2}^{2}+L_{3}^{2}} \\
M & =a b_{1}^{2}+a b_{2}^{2}+a b_{3}^{2} \\
L_{1} & =k s_{2} a b_{3}-k s_{3} a b_{2} \\
L_{2} & =k s_{3} a b_{1}-k s_{1} a b_{3} \\
L_{3} & =k s_{1} a b_{2}-k s_{2} a b_{1}
\end{aligned}
$$

The partial derivatives needed for formula (6) are expressed by the following formulae:

$$
\begin{gathered}
\frac{\partial l}{\partial a b_{1}}=\frac{\left(L_{2} \cdot k s_{3}-L_{3} \cdot k s_{2}\right)}{M \cdot L}-\frac{2 a b_{1} \cdot L}{M^{2}} \\
\frac{\partial l}{\partial a b_{2}}=\frac{\left(-L_{2} \cdot k s_{3}+L_{3} \cdot k s_{1}\right)}{M \cdot L}-\frac{2 a b_{2} \cdot L}{M^{2}} \\
\frac{\partial l}{\partial a b_{3}}=\frac{\left(L_{1} \cdot k s_{2}+L_{2} \cdot k s_{1}\right)}{M \cdot L}-\frac{2 a b_{3} \cdot L}{M^{2}} \\
\frac{\partial l}{\partial a s_{1}}=\frac{\left(-L_{2} \cdot a b_{3}+L_{3} \cdot a b_{2}\right)}{M \cdot L} \\
\frac{\partial l}{\partial a s_{2}}=\frac{\left(L_{1} \cdot a b_{3}-L_{3} \cdot a b_{1}\right)}{M \cdot L} \\
\frac{\partial l}{\partial a s_{3}}=\frac{\left(-L_{1} \cdot a b_{2}-L_{2} \cdot a b_{1}\right)}{M \cdot L}
\end{gathered}
$$

Relevant uncertainty components $u_{\mathrm{xi}}$ are: $u_{\mathrm{as} 1}, u_{\mathrm{as} 2}, u_{\mathrm{as} 3}, u_{\mathrm{ab} 1}, u_{\mathrm{ab} 2}, u_{\mathrm{ab} 3}$.

To determine what the components of the measurement uncertainty depend on, the analyses will be presented using numerical examples.

An example was examined for a connecting rod with the following dimensions: foot width $-25 \mathrm{~mm}$, head width $-20 \mathrm{~mm}$, axes distance $-120 \mathrm{~mm}$. Distribution of characteristic points was adopted as follows: $A(5,5,5)$, $B(25,5,5), K(7.5 ; 125 ; 5)$. This corresponds to the orientation of the connecting rod parallel to the $x y$ plane.

The uncertainty budget for the location of the $S$ point $(22.5 ; 125 ; 10.01)$ corresponding to the length of the toleranced element is presented in tab. I.

TABLE I. Uncertainty budget for measuring the distance of a point from a straight line (Fig. 1a) for $S$ (22.5; 125; 10.01)

\begin{tabular}{|c|c|c|c|c|}
\hline Component & $x_{i}[\mathrm{~mm}]$ & $\frac{\partial l}{\partial x_{\mathrm{i}}}$ & $u_{\mathrm{xi}}[\mu \mathrm{m}]$ & $\frac{\partial l}{\partial x_{\mathrm{i}}} u_{\mathrm{xi}}[\mu \mathrm{m}]$ \\
\hline$k s_{1}$ & 15 & 0 & 0.69 & 0 \\
\hline$k s_{2}$ & 0 & 0 & 0.67 & 0 \\
\hline$k s_{3}$ & 0.01 & 1 & 0.67 & 0.67 \\
\hline$a b_{1}$ & 20 & 0 & 0.69 & 0 \\
\hline$a b_{2}$ & 0 & 0 & 0.67 & 0.5 \\
\hline$a b_{3}$ & 0 & -0.75 & 0.67 & 0.84 \\
\hline \multicolumn{2}{|r|}{} & & &
\end{tabular}


There are two non-zero components in the uncertainty budget. One of the components, with a weight of 1 , corresponds to the distance of point $S$ from the toleranced axis $\left(k s_{3}\right)$. The uncertainty component corresponding to the $a b_{3}$ coordinates' difference occurs in the budget with a weight of 0.75 , which corresponds to the ratio of the length of the toleranced element $\left(k s_{1}=15 \mathrm{~mm}\right)$ to the length of the datum element $\left(a b_{1}=20 \mathrm{~mm}\right)$. The combined uncertainty is $0.84 \mu \mathrm{m}$.

\section{Out-of-plane parallelism of axes}

Fig. 2 shows a model for measuring out-of-plane parallelism of axes deviation.

a)

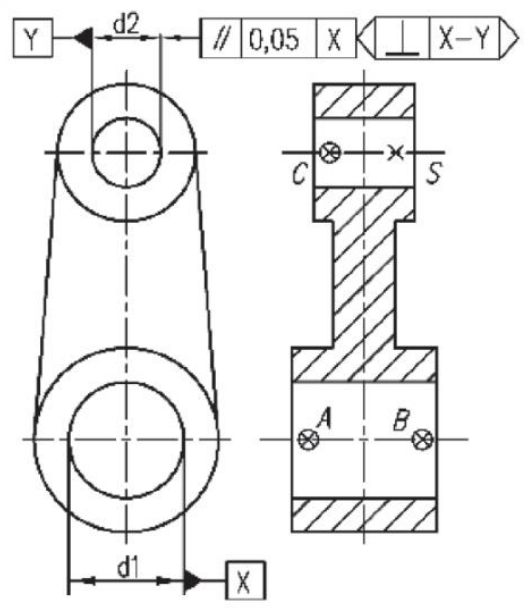

b)

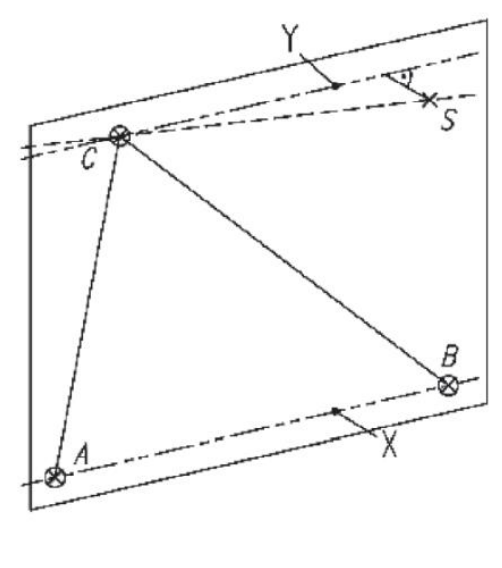

Fig. 2. Out-of-plane parallelism of axes: $a$ ) example drawing with characteristic points, $b$ ) model for uncertainty evaluation

The tolerance zone is a pair of planes parallel to the datum axis and including the toleranced axis. The parallelism deviation is the smallest distance of the pair of planes referred to in the definition of parallelism tolerance covering the actual points of the toleranced axis, i.e. distance $l$ of the point $S$ from the plane $A B C$.

Uncertainty of measurement of the out-of-plane parallelism of axes is equal to the measurement uncertainty $u_{1}$ of the distance $l$.

General formula for the distance $l$ of the point $S$ from the plane $p$, defined by the point $P$ and the unit normal vector $v$, is:

$$
l(S, p)=|(P-S) \cdot v|
$$

In the example below, the plane $p$ is represented by three points. If the plane $p$ is represented by points $A, B$ and $C$, then the unit normal vector can be defined as (Fig. 2):

$$
v=\frac{A B \times A C}{|A B \times A C|}
$$

Finally, a measurement model is obtained in the form:

$$
l(A S, A B, A C)=\left|A S \cdot \frac{A B \times A C}{|A B \times A C|}\right|
$$

Thus, the derived formula for distance $l$ (measurement model) is:

where:

$$
l=\frac{a s_{1} L_{1}+a s_{2} L_{2}+a s_{3} L_{3}}{M}
$$

$$
\begin{gathered}
L_{1}=a b_{2} a c_{3}-a b_{3} a c_{2} \\
L_{2}=a b_{3} a c_{1}-a b_{1} a c_{3} \\
L_{3}=a b_{1} a c_{2}-a b_{2} a c_{1}
\end{gathered}
$$




$$
M=\sqrt{L_{1}^{2}+L_{2}^{2}+L_{3}^{2}}
$$

Distance $l$ is here a function of nine coordinates' differences (vector components $A B, A C$ and $A S$ ), thus the combined uncertainty of measurement $u_{1}$ is the geometric sum of nine elements:

$$
u_{l}=\sqrt{\sum_{i=1}^{9}\left(\frac{\partial l}{\partial x_{i}} u_{x i}\right)^{2}}
$$

Derivatives occurring in the formula are weights (sensitivity factors), with which the uncertainties of measurement of individual distances affect the measurement uncertainty of the measured characteristic. The corresponding derivatives $\frac{\partial l}{\partial_{\mathrm{x}_{\mathrm{i}}}}$ are expressed by the formulae:

$$
\begin{gathered}
\frac{\partial l}{\partial a s_{1}}=\frac{L_{1}}{M} \\
\frac{\partial l}{\partial a s_{2}}=\frac{L_{2}}{M} \\
\frac{\partial l}{\partial a b_{1}}=\frac{L_{3}}{M} \\
\frac{\partial l}{\partial a b_{2}}=\frac{\left(-a s_{1} L_{1}-a s_{2} L_{2}-a s_{3} L_{3}\right)\left(a c_{3} L_{2}+a c_{2} L_{3}\right)}{M^{3}}+ \\
+\frac{a s_{3} a c_{2}-a s_{2} a c_{3}}{M} \\
+\frac{a s_{1} a c_{3}-a s_{3} a c_{1}}{M} \\
+\frac{\left.\partial s_{2} L_{2}-a s_{3} L_{3}\right)\left(a c_{3} L_{1}-a c_{1} L_{3}\right)}{M^{3}}
\end{gathered}
$$


Relevant component uncertainties $u_{\mathrm{xi}}$ are: $u_{\mathrm{as} 1}, u_{\mathrm{as} 2}, u_{\mathrm{as} 3}, u_{\mathrm{ab} 1}, u_{\mathrm{ab} 2}, u_{\mathrm{ab} 3}, u_{\mathrm{ac} 1}, u_{\mathrm{ac} 2}, u_{\mathrm{ac} 3}$.

An example was analysed for a workpiece with dimensions and location relative to the coordinate system as in the previous chapter: $A(5,5,5), B(25,5,5), C(7.5 ; 125 ; 5)$.

The uncertainty budget is presented in tab. II. Two components have non-zero values in it. The first non-zero component, for $C S_{3}$ with a weight of 1 , corresponds to the distance of point $S$ from the plane defined by the datum axis and the point of the toleranced axis (the parallelism of axes deviation). The second non-zero component, for $a b_{3}$ with a weight of 0.75 , corresponds to the ratio of the width of the toleranced element $\left(c s_{1}=\right.$ $15 \mathrm{~mm})$ to the width of the datum $\left(a b_{1}=20 \mathrm{~mm}\right)$.

TABLE II. Uncertainty budget for measuring the distance of a point from a straight line (Fig. $2 a$ ) for point $S(22.5 ; 125 ; 10.01)$

\begin{tabular}{|c|c|c|c|c|}
\hline Component & $x_{\mathrm{i}}[\mathrm{mm}]$ & $\frac{\partial l}{\partial x_{\mathrm{i}}}$ & $u_{\text {xi }}[\mu \mathrm{m}]$ & $\frac{\partial l}{\partial x_{\mathrm{i}}} u_{\text {xi }}[\mu \mathrm{m}]$ \\
\hline$c s_{1}$ & 15.0 & 0 & 0.69 & 0 \\
\hline$c s_{2}$ & 0.0 & 0 & 0.67 & 0 \\
\hline$c s_{3}$ & 0.01 & 1 & 0.67 & 0 \\
\hline$a b_{1}$ & 20.0 & 0 & 0.69 & 0 \\
\hline$a b_{2}$ & 0.0 & 0 & 0.67 & 0.50 \\
\hline$a b_{3}$ & 0.0 & -0.75 & 0.67 & 0 \\
\hline$a c_{1}$ & 2.5 & 0 & 0.67 & 0 \\
\hline$a c_{2}$ & 120.0 & 0 & 0.83 & 0 \\
\hline$a c_{3}$ & 0.0 & 0 & $u=$ & 0.67 \\
\hline
\end{tabular}

\section{In-plane parallelism of axes}

Fig. 3 shows a model for measuring the in-plane parallelism of axes.

The tolerance zone is a pair of planes parallel to the datum axis and perpendicular to the common plane of both axes. Parallelism deviation is the smallest distance $l$ of the pair of planes referred to in the definition of parallelism tolerance covering the actual points of the tolerance axis.

The uncertainty of measurement of the in-plane parallelism of axes is equal to the measurement uncertainty $u_{1}$ of the distance of the point from the plane.

General formula for the distance $l$ of the point $S$ from the plane $p$, defined by the point $P$ and the unit normal vector $v$, was given earlier (19). In this model, the plane $p$ contains point $K$ and is parallel to the straight line defined by points $A$ and $B$ and perpendicular to the plane $A B K$ (Fig. 3).

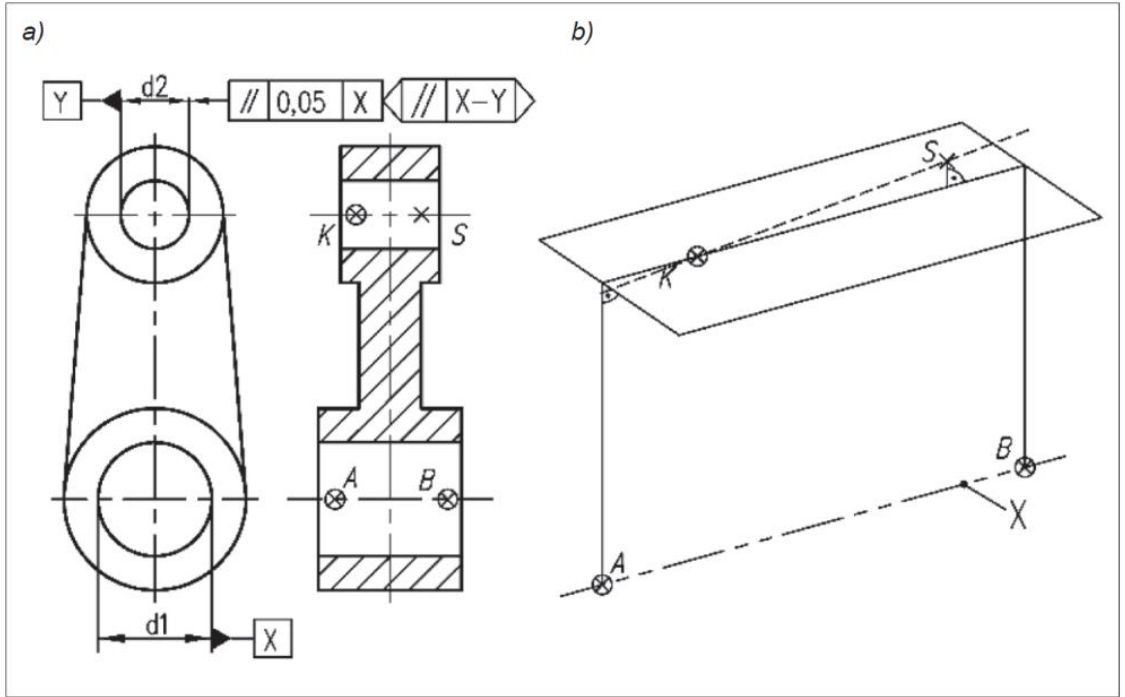

Fig. 3. In-plane parallelism of axes: $a$ ) example drawing with characteristic points, $b$ ) model for uncertainty evaluation 
Point $P$ in formula (19) is point $K$. A normal vector can be defined as the product of a vector normal to the plane $A B K$ and a vector parallel to the line $A B$. The unit normal vector of the $A B K$ plane can be defined as (Fig. 3 ):

$$
v=\frac{(A B \times A K) \times A B}{|(A B \times A K) \times A B|}
$$

TABLE III. Uncertainty budget for measuring the distance of a point from a straight line (Fig. $3 a$ ) for point $S(22.5 ; 10 ; 125)$

\begin{tabular}{|c|c|c|c|c|}
\hline Component & $x_{\mathrm{i}}[\mathrm{mm}]$ & $\frac{\partial l}{\partial x_{\mathrm{i}}}$ & $U_{\mathrm{xi}}[\mu \mathrm{m}]$ & $\frac{\partial l}{\partial x_{\mathrm{i}}} u_{\mathrm{xi}}[\mu \mathrm{m}]$ \\
\hline$a b_{1}$ & -20 & 0 & 0.69 & 0 \\
\hline$a b_{2}$ & 0 & 0 & 0.67 & 0 \\
\hline$a b_{3}$ & 0 & 0.75 & 0.67 & 0 \\
\hline$a k_{1}$ & -2.5 & 0 & 0.67 & 0 \\
\hline$a k_{2}$ & 0 & 0 & 0.67 & 0 \\
\hline$a k_{3}$ & -120 & 0 & 0.83 & 0 \\
\hline$k s_{2}$ & -15 & 0 & 0.69 & 0 \\
\hline$k s_{3}$ & 0 & 0 & 0.67 & -0.67 \\
\hline
\end{tabular}

Ultimately, the measurement model is:

$$
l(A B, A K, K S)=\left|K S \cdot \frac{(A B \times A K) \times A B}{|(A B \times A K) \times A B|}\right|
$$

An example was analysed for a workpiece with dimensions and location relative to the coordinate system as in the previous chapter $A(5,5,5), B(25,5,5), K(7.5 ; 125 ; 5)$. The uncertainty budget is presented in tab. III.

In uncertainty budget presented in tab. III, two components have non-zero values. The first non-zero component, for component $k s_{3}$ with a weight of 1 , corresponds to the distance of the point $S$ from the plane defined by the datum axis and the point of the toleranced axis (the parallelism of axes deviation). The second non-zero component, for $a b_{3}$ with a weight of 0.75 , corresponds to the ratio of the width of the toleranced element $\left(k s_{1}=15 \mathrm{~mm}\right)$ and the width of the datum $\left(a b_{1}=20 \mathrm{~mm}\right)$.

\section{Summary}

Presented measurement models reflect the essence of measurements of coordinate geometrical deviations due to the fact that they express the measurand (deviation value) as a function of directly measured quantities (coordinates' differences, i.e. values of CMM's displacements).

In the three presented cases of measurement of the parallelism of axes, identical uncertainty values were obtained (despite quite different measurement models), which can be explained by large similarity of measurement tasks and dimensions of the measured workpiece.

\section{REFERENCES}

[1] Płowucha W. „Szacowanie niepewności pomiarów współrzędnościowych metodą analizy wrażliwości - podstawy teoretyczne". Mechanik. 11 (2018): 4, https://doi.org/10.17814/mechanik.2018.11.168.

[2] Płowucha W. "Uncertainty of coordinate measurement of geometrical deviations". Procedia CIRP. 75 (2018): 361366, https://doi.org/10.1016/i.procir.2018.04.071.

[3] ISO/TS 15530-1:2013 Geometrical product specifications (GPS). Coordinate measuring machines (CMM): Technique for determining the uncertainty of measurement. Part 1: Overview and metrological characteristics.

[4] Płowucha W., Jakubiec W. „Wyznaczanie niepewności pomiarów współrzędnościowych. Cz. 3: Zastosowanie przedmiotu wzorcowego". Mechanik. 8-9 (2014): CD1, 113-121.

[5] JCGM 100:2008 "Evaluation of measurement data. Guide to the expression of uncertainty in measurement". https://ncc.nesdis.noaa.gov/documents/documentation/JCGM_100_2008_E.pdf.

[6] ISO 1101:2017 Geometrical product specifications (GPS). Geometrical tolerancing. Tolerances of form, orientation, location and run-out. 